\title{
Designing a tool for measuring the awareness of predisposing factors and dimensions of low back pain in operating room personnel and evaluating its psychometric properties
}

\section{Reza Tavakkol}

Shiraz University of Medical Sciences

Jamshid Eslami

Shiraz University of Medical Sciences

Ladan Zarshenas ( Zarshenasster@gmail.com )

Shiraz University of Medical Sciences

Azadeh Amiri

Shiraz University of Medical Sciences

Research article

Keywords: Awareness, Low back pain, Operating rooms, Psychometric, Questionnaire

Posted Date: January 27th, 2020

DOl: https://doi.org/10.21203/rs.2.21946/v1

License: (c) (1) This work is licensed under a Creative Commons Attribution 4.0 International License.

Read Full License 


\section{Designing a tool for measuring the awareness of predisposing factors and dimensions of low back pain in operating room personnel and evaluating its psychometric properties}

Reza Tavakkol ${ }^{1}$, Jamshid Eslami ${ }^{2}$, Ladan Zarshenas ${ }^{3 *}$, Azadeh Amiri ${ }^{4}$

1- Master student of Surgical Technology, Operating Room Group, School of Nursing and Midwifery, Shiraz University of Medical Science, Shiraz, Iran

2- Assistant Professor of Curriculum Planning, Department of Nursing, School of Nursing and Midwifery, Shiraz University of Medical Sciences, Shiraz, Iran

3- Associate Professor, Community-Based Psychiatric Care Research Center, School of Nursing and Midwifery, Shiraz University of Medical Sciences, Shiraz, Iran (zarshenas@sums.ac.ir)

4- MSc and Faculty Member School of Nursing and Midwifery, Operating Room Group, School of Nursing and Midwifery, Shiraz University of Medical Science, Shiraz, Iran

*Correspondence: Dr Ladan Zarshenas, Department of Mental Health and Psychiatric Nursing, Community Based Nursing and Midwifery Research Center, School of Nursing and Midwifery, Shiraz University of Medical Sciences, Shiraz, Iran. Email:zarshenas@ sums.ac.ir P.O.Box:71936-13119

Tel: +98 711-6474255,6474250-197- 228 


\title{
Designing a tool for measuring the awareness of predisposing factors and dimensions of low back pain in operating room personnel and evaluating its psychometric properties
}

\begin{abstract}
Background: Low back pain is the most common type of work-related musculoskeletal disorders and one of the costliest health problems. This study aimed to design a psychometric tool to measure the operating room personnel's awareness of predisposing factors and dimensions of low back pain.

Methods: This methodological study aimed to design a psychometric tool for assessing the operating room personnel's awareness of predisposing factors and dimensions of low back pain in. In the first step, the main dimensions and concepts were developed by reviewing the literature and consulting with professors and experts. In the second step, the initial items of the questionnaire were formulated. Finally, the psychometric properties of the questionnaire were determined using face, content and construct validity, internal consistency, and test-retest reliability.
\end{abstract}

Results: In the first phase of the study, the concept of low back pain and its risk factors were determined, and then the initial items of the tool were developed. In the next step, face and content validity of the instrument were determined both qualitatively and quantitatively (content validity ratio $=0.91$ and content validity index $=0.95)$. Indeed, construct validity was assessed by convergent and divergent validity using chronic pain and general health questionnaires, respectively (correlation coefficient in convergent validity $=0.70$ and correlation coefficient in divergent validity=-0.37). Finally, the reliability of the instrument was determined using internal consistency $(\alpha=0.60)$ and test-retest reliability (0.77).

Conclusion: Considering the design, validity, and reliability of the instrument for measuring the awareness of predisposing factors and dimensions of low back pain in operating room personnel, we suggest that it should be used for evaluation of the awareness and different dimensions of low back pain among the operating room personnel.

Keywords: Awareness, Low back pain, Operating rooms, Psychometric, Questionnaire 


\section{Introduction:}

Low back pain is the most common type of work-related musculoskeletal disorders and one of the costliest health problems $(1,2)$ that causes a significant decrease in the quality of services and absence from work (3). Low back pain can lead to activity limitations and diseases in more than $50 \%$ of nurses(4). It is also the second most common diagnosis in the United States(5). Indeed, it has been shown to cause a higher average life-long disability compared to AIDS, road injuries, tuberculosis, lung cancer, chronic obstructive pulmonary disease, and complications of preterm labor(6). The prevalence of low back pain has been estimated to be $15-45 \%$ among the general population worldwide. Besides, it has been estimated that approximately $70-80 \%$ of the general population experience a back problem some time in their lives $(4,7,8)$. The prevalence of low back pain, as the most important musculoskeletal disorder, has been reported to be $30-60 \%$ among nurses(9). In Iran, the prevalence of low back pain has been reported to be $63 \%$ amongst the nurses(10). Healthcare workers are at risk of work-related low back pain due to physical and emotional factors and often experience more low back pain in comparison to construction workers, miners, and those working in factories $(11,12)$. Among healthcare workers, the highest prevalence of low back pain (40-50\% annually) has been detected in nurses and operating room personnel(4). The prevalence of low back pain has been reported to be $50.3 \%$ among operation room personnel in $\operatorname{Iran}(10)$. The operation room affects individuals both physically and psychologically(13). In addition, considering the hazardous activities performed in the operating room, low back pain is a common health problem among these personnel(4). The risk factors that can cause discomfort and musculoskeletal disorders among the operatinon room personnel include static stress (such as long-standing, constant physical posture, and holding 
equipment such as retractors during surgery), and manual handling (such as pulling, pushing, or lifting tools and heavy equipment)(13). Hence, operating room personnel have the highest prevalence of low back pain due to unpleasant physical conditions during surgery, movement restriction during surgery, and stress and burnout resulting from night work(14). Prolonged standing and working constantly in standing position can lead to cardiovascular problems, back pain, leg pain and swelling, and reduction of blood flow to muscles, eventually causing general muscle fatigue(15). Musculoskeletal disorders, especially low back pain, may be significantly reduced by careful observation of the principles of ergonomics in the workplace, regular physical examinations, identification of the risk factors, and regular exercise and physical activity(16). Since low back pain has been introduced as the most important reason for burnout among the operation room personnel(17), increasing the personnel's awareness of the predisposing factors of low back pain is expected to reduce the high prevalence of this disorder and prevent its subsequent complications.

Based on what was mentioned above and the studies conducted on the issue, low back pain is one of the most important occupational injuries amongst the operation room personnel and one of the factors that can reduce staffing in this profession. Nonetheless, an extensive review of the literature revealed no specific tools to measure the awareness of the predisposing factors of low back pain among the operatinon room personnel. The existing tools have often been designed to measure the prevalence and risk factors of low back pain among medical personnel, specifically nurses. Indeed, there is no standardized tool to assess the level of awareness of the risk factors of low back pain. Considering the occupational differences between the operation room personnel and nurses and other medical units and lack of any specific tools in this field, the present study 
aims to design and psychometrically evaluate an instrument for this group of medical staff.

\section{Methods:}

Procedure

The present methodological research was conducted at Shiraz University of Medical Sciences in 2019. In the first step, the main dimensions and concepts of the issue were developed through an extensive review of the literature and consultation with professors and experts in the fields of operating room, musculoskeletal disorders, and pain. In the second step, the initial items were examined and developed. Thus, the initial format of the tool was designed to assess the awareness of the predisposing factors and dimensions of low back pain based on the Likert scale (yes, no, I do not know). In the last step, the psychometric properties of the instrument were evaluated using various methods.

Psychometric evaluation

After designing the initial format of the tool, its psychometric evaluation steps began. Generally, the psychometric properties of an instrument are assessed in order to determine how it measures the intended purpose. In the current study, qualitative and quantitative methods were used to determine face, content, and construct validity.

Cross-sectional Validity

The qualitative face validity was assessed through face-to-face interviews with 20 operation room personnel and professors. This was done to investigate the difficulty of the items, ambiguity, and proportion as well as their relevance to the main purpose of the study. Considering the quantitative face validity, 20 operation 
room personnel with bachelor's degrees were asked to evaluate the initial questionnaire regarding the importance of each item using the quantitative item impact method. Then, the impact score of each item was calculated separately based on the following formula. In this way, a five-point Likert scale was considered for each item as follows: it is quite important (score 5), it is somewhat important (score 4), it is moderately important (score 3), it is slightly important (score 2), and it is not important at all (score 1).

\section{Impact Score $=$ Frequency $(\%) \times$ Significance}

In case the impact score of an item exceeded 1.5, it was considered appropriate for the subsequent analyses and retained(18). In general, content validity is assessed to ensure that the content of a test represents the construct it claims to measure. It also aims to determine whether the content represents the set of the properties being measured(19). In the present study, qualitative and quantitative methods were used to determine the content validity of the questionnaire. Because assessment of content validity at this stage was based on the experts' judgments, their opinions in the areas of tool design, pain, and operation room were used. In the qualitative content validity, the experts were asked to present their viewpoints concerning grammar, wording, location of items, and scoring. Afterwards, Content Validity Ratio (CVR) and Content Validity Index (CVI) were used in order to evaluate the quantitative content validity. CVR aimed to explore the necessity of an item from the experts' viewpoints, while CVI aimed at determining the relevance of the item to the study objectives. In order to determine CVR, we asked 14 experts to respond to each item based on a three-point Likert scale as follows: "necessary, useful but not necessary, and not necessary". Then, the responses were evaluated according to the following formula and Lawsche table for 14 people with a minimum of 0.51 . 
After calculating CVR for all items, those with a score of at least 0.51 were retained for the next step and those with a score less than 0.51 were excluded(20) $C V R=\frac{n_{E}-\frac{N}{r}}{\frac{N}{r}}$

Waltz and Bausell's method was used to evaluate CVI. In doing so, the tool was given to 10 experts and they were asked to rate the relevance, clarity, and simplicity of each item based on a 4-point Likert scale (scores 1 to 4). Then, CVI was computed using the following formula(19)

$$
\mathrm{CVI}=\sum \frac{\sum \text { number of answers } 3 \text { or } 4}{\text { Total Number of answers }}
$$

After calculating CVI based on the total number of agreed points for each item (scores three and four in the four-point scale), scores above 0.79 were considered to be suitable and acceptable, scores between 0.70 and 0.79 were regarded as questionable and requiring correction, and scores less than 0.70 were determined as unacceptable(21).

Internal Structure: Construct validity

Considering convergent and divergent construct validity, the Chronic Pain Questionnaire was used as the convergent instrument and General Health Questionnaire, the validity and reliability of which were investigated by Seyed Mohammad Reza Taghavi(22) was used as the divergent tool. At this stage, the questionnaires were completed by 20 operation room personnel selected randomly. In the end, the correlation between these questionnaires and the designed instrument was explored. 
Internal Consistency: Reliability

Reliability is associated with stability and repeatability. In general, reliability allows us to estimate the proportion of error diffraction that constitutes the diffraction of the whole test. Using a reliable instrument repeatedly, the scores are not affected by the random error. Nonetheless, no tool is completely reliable even in the most desirable conditions(19). In this study, test-retest and internal consistency methods were used to evaluate the reliability of the instrument. These methods answer the question whether a person's score on a test remains constant over time. In other words, when a group is tested and retested after a certain time, a high correlation is expected between the scores of the two tests. Considering testretest reliability in the present study, the questionnaire was distributed among 20 randomly selected operation room personnel. Then, the questionnaire was given to the same group after two weeks. Intra-cluster correlation was determined by comparing and analyzing the results (19). Cronbach's alpha was also used to measure the internal consistency of the instrument. Generally, Cronbach's alpha should be between 0.7 and 0.8 for a good correlation. In this study, internal consistency was determined by selecting 20 operation room personnel randomly. In this way, the items were examined in terms of concept suitability. The alpha coefficient is the most widely used statistical method to determine the internal consistency and it is the only indicator of reliability that requires only one test run. In this method, the correlation between each item and other items is important. The alpha coefficient was calculated by taking the mean of item-to-item correlations by using the following formula $(19,21)$

$a=\frac{N \mathrm{p}}{[1+\mathrm{P}(\mathrm{N}-1)]}$ 


\section{Ethical considerations}

In this study, all ethical points were observed and all steps were performed with the permission of the Ethics Committee of Shiraz University of Medical Sciences (code of ethics: IR.SUMS.REC.1398.263). Before beginning the study, its goals were explained to the participants and their written informed consents were obtained. The participants were informed that participation in the study was voluntary and they were allowed to withdraw at any time. They were also reassured about the anonymity of the publications.

\section{Results:}

During the first phase of the research, by reviewing the existing texts and consulting with the professors, 29 items were obtained for the tool. In this way, the first format of the tool was made and entered into the face validation phase.

\section{Validity}

This phase of psychometric evaluation was done in both qualitative and quantitative forms. Assessment of the qualitative face validity led to the modification of three items. Indeed, two items were added by the experts at the end of this phase. Thus, 30 items were entered into the quantitative face validity phase. In this phase that assessed the importance of each item by using the item impact formula, all 31 items obtained the minimum impact score, i.e. 1.5, and were then entered into the next phase of psychometric evaluation.

Qualitative and quantitative methods were used to assess the content validity of the instrument. Assessment of the qualitative content validity resulted in modification of three items and integration of one item into other items, eventually leading to 30 items in the next phase of psychometric evaluation. Quantitative content validity was evaluated using both CVR and CVI. Based on the Lawsche table, the 
minimum CVR for the 14 experts was higher than 0.51 , and only one item did not receive the required minimum score and was omitted. The mean CVR of the items was 0.91. Thus, 29 items were entered into the CVI calculation phase. Based on the investigation of CVI according to Waltz and Bausell's method by 10 experts, scores above 0.79 were recommended for item acceptance. Accordingly, only one item scored less than 0.79 and was excluded. The mean CVI of the items was 0.95. At the end of this phase, the 28 items that had received the required scores were entered into the next phase of psychometric evaluation.

\section{Construct validity}

In order to evaluate the convergent and divergent validity, the correlation between the designed instrument and Chronic Pain Questionnaire and General Health Questionnaire was explored in 30 operation room personnel. The results showed a significantly high correlation (0.702) between the designed instrument and the Chronic Pain Questionnaire as the convergent instrument $(\mathrm{p}=0.035)$. A significant correlation (-0.375) was also observed between the designed instrument and the General Health Questionnaire as the divergent tool $(\mathrm{p}=0.041)$.

\section{Reliability}

The reliability of the tool was evaluated by using internal consistency and testretest methods. The internal consistency was assessed via measurement of Cronbach's alpha among 20 operation room personnel. After analyzing the questionnaires, Cronbach's alpha was computed as 0.608, which indicated the reliability of the instrument. The test-retest method was also performed on 20 operation room personnel with a two-week interval. After comparing and analyzing the scores using the intra-cluster correlation index during these two stages, the correlation coefficient was computed as 0.77 , which was statistically 
high and significant $(\mathrm{p}<0.001)$. This indicated that the answers to the questions were very similar in the two stages of the test.

\section{Discussion:}

This study aimed to design a tool to measure the operation room personnel's awareness of the predisposing factors and dimensions of low back pain and to evaluate its psychometric properties. This questionnaire was designed based on the factors affecting the incidence of low back pain and operating room conditions followed by investigation of face validity, content validity, construct validity, and reliability. The questionnaire consisted of two parts. The first part evaluated low back pain via 10 multiple-choice questions, while the second part assessed the awareness of low back pain by 17 items using a three-point Likert scale (yes, no, I do not know).

Lawlis et al. conducted a study in 1989 and designed the Dallas Pain Questionnaire to assess the effect of spinal pain on the patients' behaviors. In order to assess the psychometric properties of this questionnaire, they made use of factor analysis. They also evaluated convergent validity by using McGill Pain Questionnaire in order to assess the construct validity. Finally, they tested the reliability of the tool through the test-retest method. The results showed that the correlation coefficient was equal to 0.97 , which indicated great similarity between the responses in both test and retest(23).The differences between the Dallas Pain Questionnaire and the instrument designed in the present study could be attributed to the differences between the target groups (the present study focused on the operation room personnel) as well as the psychometric assessment stages involving quantitative and qualitative face and content validity methods and determination of construct validity by calculating the correlation coefficient in convergent and divergent validities. 
In order to assess the reliability of the questionnaire in the current study, we calculated Cronbach's alpha coefficient (0.608) in addition to the test-retest method (correlation coefficient=0.77), indicating the high accuracy of the study.

Anagnostis et al. determined the psychometric properties of the Pain Disability Questionnaire in 2004. In that study, construct validity was assessed by factor analysis and convergent validity using the Oswestry Questionnaire. The results revealed a significant correlation between the two instruments. Moreover, the reliability of the tool was assessed by the test-retest method, demonstrating the correlation coefficient of 0.97 . Internal consistency of the instrument was also approved by Cronbach's alpha coefficient of 0.96(24). The correlation between the scores in the test-retest method and the Cronbach's alpha coefficient were higher in the study performed by Anagostis in comparison to the instrument designed in the present study. This might be associated with working in the stressful conditions of the operation room and its impacts on the personnel's bodies and minds.

In another study in 2005 , Kim et al. evaluated the validity and reliability of a questionnaire on the impotence created by spinal diseases in Korea. In order to determine the construct validity of the instrument, we assessed convergent validity using the World Health Organization's Quality of Life Questionnaire, indicating the correlation coefficient of 0.48 . Its reliability was also assessed by internal consistency method (Cronbach's alpha of 0.84) and test-retest method with a twoday interval (correlation coefficient of 0.91)(25). One of the strong points of the current study was that both convergent and divergent validity were used to determine the construct validity of the designed instrument, while only convergent validity was used in Kim's study. Similar to Kim's study, reliability was assessed via internal consistency and test-retest methods, but the only difference was that the test-retest method involved a two-week interval in the present study. 
Manouchehr Shirazi et al. carried out a research to design and psychometrically evaluate the Chronic Pain Acceptance Questionnaire in the elderly individuals in 2014. The validity of the instrument was assessed through face validity, content validity, and construct validity by using exploratory factor analysis. The results showed that the means of CVR and CVI were 0.94 and 0.92 , respectively. Additionally, the reliability of the instrument was determined by the internal consistency (Cronbach's alpha of 0.83) and test-retest methods (correlation coefficient of 0.85)(26). The psychometric evaluation stages in Shirazi's study were similar to those of the present study, except for the fact that convergent and divergent validity were used to explore construct validity in the current study. Also, the means of CVR and CVI were 0.91 and 0.95 , respectively in the present survey, which showed no significant differences with the measures reported in Shirazi’s study.

Zarshenas et al. conducted a study in 2018 to design a questionnaire for measuring the health needs of patients with chronic low back pain and explore its psychometric features. Psychometric evaluation was done through face validity (qualitative and quantitative), content validity (qualitative and quantitative), and construct validity through exploratory factor analysis. Besides, internal consistency method was used to test the reliability, which revealed the Cronbach's alpha coefficient of $0.70(8)$. The psychometric evaluation stages in that study were the same as those used in the present study, except for the construct validity. Factor analysis was used to evaluate the construct validity in the study by Zarshenas et al., while convergent and divergent validity were employed in the present study. Furthermore, reliability was assessed via the internal consistency method and calculating the alpha coefficient in the study by Zarshenas et al., while the testretest method was also used in the current study. 


\section{Conclusion:}

The present questionnaire was designed for assessment of the awareness of predisposing factors and dimensions of low back pain among healthcare team members, particularly operation room personnel, through an intensive investigation of credible scientific sources in the field of low back pain. Considering its appropriateness for assessing the awareness of predisposing factors and dimensions of low back pain, suitable validity and reliability, simple scoring, ease of use, comprehensiveness, and unavailability of similar questionnaires, it is recommended that this questionnaire should be used to evaluate the operation room personnel.
Abbreviations
CVR, Content Validity Ratio; CVI, Content Validity Index

\section{Declarations}

\section{Ethical approval and consent to participate}

Written informed consents were obtained from all participants. The study was approved by the Ethics Committee of Shiraz University of Medical Sciences (IR.SUMS.REC.1398.263).

\section{Consent to publish}

Not applicable.

\section{Availability of data and materials}


The datasets during the current study are not publicly available due to confidentiality of the students' data, but they will be available upon reasonable request.

\section{Competing interests}

The authors declare that they have no competing interests.

\section{Funding}

Not applicable.

\section{Author's contributions}

J.E., L.Z. and A.A participated in study design, data collection, and data analysis. R.T. participated in study design, data collection, and data analysis. All authors read and approved the final manuscript.

Acknowledgements: The authors would like to thank Shiraz University of Medical Sciences, Shiraz, Iran and also Center for Development of Clinical Research of Nemazee Hospital and Dr. Nasrin Shokrpour for editorial assistance. 


\section{References:}

1. Mekonnen TH. Work-Related Factors Associated with Low Back Pain Among Nurse Professionals in East and West Wollega Zones, Western Ethiopia, 2017: A Cross-Sectional Study. Pain and therapy. 2019:1-9.

2. Choobineh A, Taghirpour-kazerooni M, Tabatabai S, Kamalinia M. Study of nurses' knowledge on low back pain risk factors in Hospitals of Shiraz University of Medical Sciences. Journal of Health \& Safety at Work. 2012;2(1):55-62.

3. Qareeballa AA, Alhamdan OA, Almutawaa AA, Alsayed IM, Kamal FA, Al Abdrabbuh DS, et al. Prevalence of low back pain among female nurses working in secondary and tertiary healthcare, kingdom of Bahrain. International Journal of Medical Science and Public Health. 2018;7(3):183-8.

4. Homaid MB, Abdelmoety D, Alshareef W, Alghamdi A, Alhozali F, Alfahmi N, et al. Prevalence and risk factors of low back pain among operation room staff at a Tertiary Care Center, Makkah, Saudi Arabia: a cross-sectional study. Annals of occupational and environmental medicine. 2016;28(1):1. 5. Starkweather AR, Ramesh D, Lyon DE, Siangphorn U, Deng X, Sturgill J, et al. Acute low back pain: differential somatosensory function and gene expression compared to healthy no-pain controls. The Clinical journal of pain. 2016;32(11):933.

6. Awosan KJ, Yikawe SS, Oche OM, Oboirien M. Prevalence, perception and correlates of low back pain among healthcare workers in tertiary health institutions in Sokoto, Nigeria. Ghana medical journal. 2017;51(4):164-74.

7. Richardson A, McNoe B, Derrett S, Harcombe H. Interventions to prevent and reduce the impact of musculoskeletal injuries among nurses: A systematic review. International journal of nursing studies. 2018;82:58-67. 
8. Rostami K, Sharif F, Zarshenas L, Ebadi A, Farbood A. Health Needs in Patients Suffering from Chronic Back Pain: A Qualitative Study. Anesthesiology and pain medicine. 2019;9(2).

9. Moreira RF, Sato TO, Foltran FA, Silva LC, Coury HJ. Prevalence of musculoskeletal symptoms in hospital nurse technicians and licensed practical nurses: associations with demographic factors. Brazilian journal of physical therapy. 2014;18(4):323-33.

10. Azizpour Y, Delpisheh A, Montazeri Z, Sayehmiri K. Prevalence of low back pain in Iranian nurses: a systematic review and meta-analysis. BMC nursing. 2017;16(1):50.

11. Wong $\mathrm{T}$, Teo N, Kyaw M. Prevalence and risk factors associated with low back among health care providers in a District Hospital. Malaysian Orthopaedic Journal. 2010;4(2):23-8.

12. Johnson OE, Edward E. Prevalence and risk factors of low back pain among workers in a health facility in South-South Nigeria. British Journal of Medicine and Medical Research. 2016;11(8).

13. Choobineh A, Movahed M, Tabatabaie SH, Kumashiro M. Perceived demands and musculoskeletal disorders in operating room nurses of Shiraz city hospitals. Industrial health. 2010;48(1):74-84.

14. Attar SM. Frequency and risk factors of musculoskeletal pain in nurses at a tertiary centre in Jeddah, Saudi Arabia: a cross sectional study. BMC research notes. 2014;7(1):61.

15. Waters TR, Dick RB. Evidence of health risks associated with prolonged standing at work and intervention effectiveness. Rehabilitation Nursing. 2015;40(3):148-65.

16. Samaei SE, Mostafaee M, Jafarpoor H, Hosseinabadi MB. Effects of patient-handling and individual factors on the prevalence of low back pain among nursing personnel. Work. 2017;56(4):55161.

17. Phillips N. Berry \& Kohn's operating room technique: Elsevier Health Sciences; 2016.

18. Grossman JAC, Payne EK. A randomized comparison study regarding the impact of shortduration, high-intensity exercise and traditional exercise on anthropometric and body composition measurement changes in post-menopausal women-A pilot study. Post reproductive health. 2016;22(1):14-9.

19. Ebadi A, Zarshenas L, Rakhshan M, Zareiyan A, Sharifnia S, Mojahedi M. Principles of scale development in health science. Tehran: Jame-e-negar. 2017.

20. Polit DF, Beck CT, Owen SV. Is the CVI an acceptable indicator of content validity? Appraisal and recommendations. Research in nursing \& health. 2007;30(4):459-67.

21. Rostami K, Sharif F, Zarshenas L, Ebadi A, Farbood A. Design and psychometrics of measurement tool of health needs in patients with chronic back ache. Revista Latinoamericana de Hipertension. 2018;13(3):277.

22. Taghavi S. Validity and reliability of the general health questionnaire (ghq-28) in college students of shiraz university. Journal of psychology. 2002;5(4):381-98.

23. Lawlis GF, Cuencas R, Selby D, McCoy CE. The development of the Dallas Pain Questionnaire. An assessment of the impact of spinal pain on behavior. Spine (Phila Pa 1976). 1989;14(5):511-6.

24. Anagnostis C, Gatchel RJ, Mayer TG. The pain disability questionnaire: a new psychometrically sound measure for chronic musculoskeletal disorders. Spine. 2004;29(20):2290-302.

25. Kim D-Y, Lee S-H, Lee H-Y, Lee H-J, Chang S-B, Chung S-K, et al. Validation of the Korean version of the oswestry disability index. Spine. 2005;30(5):E123-E7.

26. Shirazi M, Manoochehri H, Zagheri Tafreshi M, Zayeri F. Development and psychometric evaluation of chronic pain acceptance instrument in the elderly. Anesthesiology and Pain. 2015;5(4):3347. 